\title{
REACTION DYNAMICS IN AN ERGODIC SYSTEM: THE SIAMESE STADIUM BILLIARD*
}

\author{
Nelson DE LEON** and B.J. BERNE \\ Department of Chemustry, Columbıa Unuerstly, New York, New York 10027, USA
}

Recurved 17 May 1982, in linal form 7 September 1982

To study the roles of chaos in energy transfer and reaction dy namics in isolated moleculcs, we devise a simple classical dynamical system with measure preserving ergodic flow. Although the dynamical system is completely chaolic, it is found that dy namical correlations give rise to substuntıl deviations from RRKM theory.

\section{Introduction}

To understand how chemical rate laws and rate constants emerge from molecular dynamics we have studied the classical dynamics of simple hamiltonian systems consisting of between two and twelve degrees of freedom $[1,2]$. These systems were all capable of undergoing geometrical isomerization, and the transition from one isomier to another involved passage over potential barriers. Barner crossing requires energy transfer between the reactive coordmate and the other molecular internal degrees of freedom. This process becomes random only if there is a transition from quasıperiodic motion (KAM regime) to chaotic motion involving reactive trajectones. Thus the reacIIon process must be studied in the light of modern developments in non-linear dynamics $[3,4]$.

In our previous papers $[1,2]$ we studied non-ergodic hamiltonian systems. In this paper we focus on reacton dynamics in an ergodic hamultonian system; particularly with respect to the conditions required for RRKM behavior. First we devise a sumple dynamical system with measure invariant ergodic flow that undergoes geometncal isomerization. Since this system is constructed from the stadium billard [3], we call It the Siamese stadium billiard. The system is a $K$ system - meaning that trajectories starting from

\footnotetext{
- Supported by a grant trom the National Seience Toundation

* I'resent address. Los Alamos National Laboratory, Los Alumos, New Mevico 87545, USA
}

neiglboring points in phase space, no matter how close - separate exponentially in time [4]. This spreading in phase space occurs with a rate constant given by the maximal Lyapunov exponent $\lambda$ or equivalently the $[5,6]$ Kolmogorov entropy $h$. By studying tume correlation functions, and particularly the reactive flux, it is possible to explore under what conditions unimolecular rate laws and rate constants exist, and moreover when RRKM theory is valid $[1,2$, 7]. In this paper we show that the reaction dynamics can be correlated with the Kolmogorov entropy (or equivalently the Lyapunov exponent). We show that deviations from RRKM behavior occur when the flow can recross the transition state in a time short compared to the mixing time $\tau_{M}$, where $\tau_{M}$ is proportonal to $\lambda^{-1}$ (or equivalently to $h^{-1}$ ). This leads to results that at first sight appear to be paradoxical. For example, for energies very close to the barrier height, It might be expected that the system will get trapped in a well for times very long compared to the mixing time $\lambda^{-1}$ so that by the time the particle recrosses the barrier it has equilibrated in the well. This would lead one to expect RRKM theory to be valid. Quite the contrary is observed, and it is easy to understand why this takes place.

In a subsequent paper [8] we study a generalization of the model for which the phase space decomposes into regular and irregular regions. 


\section{Reaction dynamics in the Siamese stadium}

The Sumese stadium bulliard (SSB) consists of a point mass, $m=1$, moving in a planc convex region with differentiable border (except for the hole) shown in fig. $1 \mathrm{a}$.

The billard moves freely inside the region, but suffers elastic collisions with the boundary. The particle can pass through the hole only if $m x^{2} / 2>E_{0}$, otherwise it is elastically scattered from the hole. This leads to activated barrier crossing. For simplicity we study the dynamics for fixed $m=1, E=1, R=1$. Since barrier crossing can take place only if $E>E_{0}$, it follows that $E_{0}<1$.

The stadium billiard (SB) defined in fig. Ib has played a prominent role in several recent studies [3]. While the circular billard, $\gamma=0$, is completely integrable, the stadium billard, $\gamma>0$, was proved by Bunimovich [9] to be a $K$ flow (cf. fig. 1 for a definltion of $\gamma$ ). Such systems are stochastic and ergodic. It is possible to map each trajectory of the Siamese stadium bullard (SSB) onto a particular trajectory of the stadium billiard (SB) by simply replacing $-x$ by $+x$ whenever $x<0$ in the Siamese stadium. It follows that the Siamese stadium is also a $K$ flow.

In a very lucid and exhaustive study of the stadium bullıard (fig. 1b), Bennettin and Strelcyn [5] computed
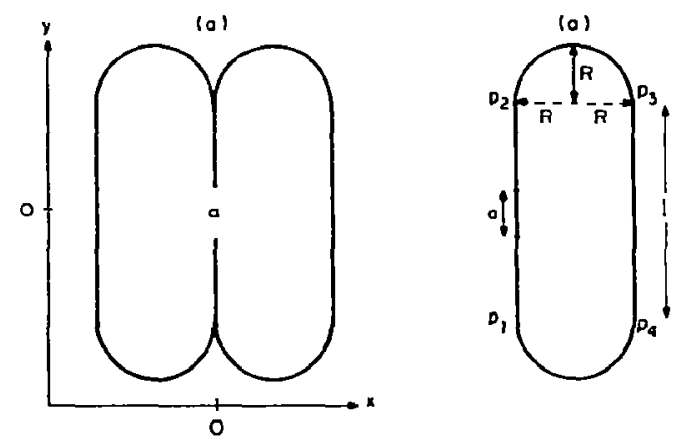

Fy 1. (a) The Siamese stadium. (b) The stadium billard consists of a unit pount mass moving freely inside the bound ary and elastically scattering off the walls The boundary consists of two semicurcles of radius $R$ joined contunuously at pounts $P_{1}, P_{2}, P_{2}, P_{3}$ and $P_{4}$ to parallel walls of length $l$, separated by a distance $2 /$ The hole in the Siamese stadium is of length a. The parameters refered to in the text are $\kappa=a / l, \gamma=l / 2 R$, the energy barner $E_{0}$, and the total energy $E$. In all studies $R=1.0$ and $E=1.0$. the maximum Lyapunov characteristic number, $\lambda$, for a series of stadium bulliards with $0<\gamma \leqslant 10$. These results are summarized in fig. 4 of ref. [3]. $\lambda$ gives the rate at which trajectories starting at neighboring points in phase space, no matter how close, exponentially separate, and can thus be thought of as a mining rate Bennettin and Strelcyn [5] could find no variation of $\lambda$ with the intial state. It follows from this that the Kolmogorov entropy $h$ is identical to $\lambda$. At $\gamma=0, \lambda=$ 0 (as expected for a regular system). $\lambda$ increases rapidly with $\gamma$, reaches a maxumum at $\gamma \approx 1$ and then decreases very slowly with $\gamma$. Thus by varying the anisotropy, $\gamma$, it is possible to study systems as a function of mixing rate, $\lambda$ (or mixing tume $\tau_{M} \equiv \lambda^{-1}$ ). It is umportant to recognize that two stadiums with the samc $\gamma$ but different energy $E$, mass $i n$ and area $A$, have different Lyaponov exponents, but these scale as $\lambda\left(\gamma, m^{\prime}, A^{\prime}, E^{\prime}\right)=\lambda(\gamma, m, A, E)\left(E^{\prime} A m / L A^{\prime} m^{\prime}\right)^{1 / 2}$,

where the area of a stadium is given by

$A=A(l / 2, r)=(4 \gamma+\pi) R^{2}$.

Bennettu and Strelcyn's fig. 4 corresponds to a series of stadiums with $A=A(1,1), E=1 / 2$ and $m=1$.

Molecular dynamics in the Siamese stadium can be generated from a study of the stadium. If one samples trajectories from a microcanonical ensemble, then for every trajectory crossing the boundary from, say, left to right, there will be an equivalent trajectory passing from right to left. Therefore, dynamics in the Siamese stadıum is equivalent to "tagging" trajectories in the ordinary stadium each time they hit and elastically reflect off the transition state (TS) region a. If we label the trajectory by $\sigma=-1$ or 1 depending on whether it would pass through the TS or not, then in addition to recording $r$ and $p$ we must also record $\sigma$. Needless to say, whether or not the trajectory passes through the hole depends on whether or not it satusfies $\dot{x}^{2}>2 E_{0}$. Hence we need only consider dynamics in the ordinary stadium when determuning averages over the microcanonical ensemble. It follows from the foregoing that the Lyaponov exponent $\lambda$ for the Slamese stadium is identical to that of the stadium.

To study reaction dynamics in the SSB it is useful to determine the reactive flux $[1,2]$ 
$k(t ; E) \equiv\left(X_{A} X_{\mathrm{B}}\right)^{-1}(\dot{x}(0) \delta(x(0)) \theta(x(t)))_{E}$,

where $X_{\mathrm{A}}=X_{\mathrm{B}}=1 / 2$ is the fraction of time spent in the wells $A$ and $B$ (cf fig. $1 b$ ), $x$ is the reaction coordinate, $\dot{x}(0) \delta(x(0))$ is the incident flux through the $\mathrm{TS}$, and $\theta(x(t))$ is the characteristic function that measures whether the trajectory is in the right well at tinie $t$ Its time dependence is recorded through the tagging process discussed previnusly. Eq (3) is evalunted using a microcanonical ensemble average at energy $E$. The delta function requires the system to be intially at the transition state $x=0$ if $k(t, E)$ decays exponentualy (at long tume), then the decay rate $\tau_{R=1}^{-1}$ is the kinetıc rate constant (the sum of the forward and backward rate constants). The RRKMl rate constant is found from the $t \rightarrow 0+$ limit of $k(t . L)\{1,7\}$. This gives

$\tau_{R K K M}^{-1}=\left(X_{A} X_{B}\right)^{-1}\langle\dot{x} \delta(x) O(\dot{x})\rangle_{E}$.

It is thus convenient to define the nomalized (dimensionless) reactive flux, $\hat{k}(t) \equiv k(\varepsilon, E) \tau_{\mathrm{RRKM}}$. Evaluation of this for the Siamese stadium bulliard gives

$T_{\text {RRK } U}^{-1}=(4 a / A)\left[\left(E-E_{0}\right) / 2\right]^{1 / 2} \theta\left(E-E_{0}\right)$.

Eq (3) is cvaluated using the procedure outlned in appendix $B$ of ref. [2]. The sampling procedure is used to choose inttal states in the $S B$, and the tagged trajectories in the $\mathrm{SB}$ are used to determine $\hat{k}(t, E)$.

\section{Results}

Several systems were studied. Each system is defined by the parameters given in fig. 1a. The parameter $K^{\prime}(\equiv a / l)$ defines the size of the TS, $a$, relative to the length, $l$, of the straght side of the stadium. The parameter $\gamma(\equiv l / 2 R)$ gives the length, $l$, to width, $2 R$, ratio of the single stadum from which the Siamese stadium is composed. As indicated in fig. 1 the total energy $E$, the mass of the billiard $m$, and the radius $r$, are fixed at $E=1, m=1$, and $r=1$ for all systems studled The only other parameter considered is the barner height, $E_{0}<1$, that determunes whether or not the particle can pass through the hole.

In section 2 it was mentioned that the initial value of the reaction flux is the RKKM rate constant $\tau_{\text {RRKM }}^{-1}$. The normalized reactive flux $\hat{k}(t)$ corresponding to $E_{0}=0.45, \kappa=0.02, \gamma=05$ is presented in fig. $2 \mathrm{Al}$. Fig. 2A2 gives the logarithmic plot versus time. The flux $\hat{k}(t)$ decays exponentially in tume with a decay constant $\tau_{R \times n}^{-1}$ which is found to be equal to $\tau_{R R K M l}^{-1}$, the RRKMI rate constant. Sunce $\tau_{\text {RRKM }}$ gives an approximate measure of the tume spent by a trajectory trapped in either the A or B stadum (cf. Iig. la), and since $\lambda^{-1}$ gives a measure of the mixing tume or correlation time of the trajectories, the quantity $\lambda \tau_{\text {RRKM }}$ gives a measure of the number of independent mixing times spent by a trajectory while it is trapped in a well. If $\lambda \tau_{\text {RRKM }}$ is large, we expect that the system will have a chance to equipartition in a well before it has a chance to recross the hole. Using $\lambda$ determined by Bennettin and Strelcyn $[3](E=2, m=1, \gamma=0.5)$, properly transformed using eq. (1) to our system ( $E=$ $1, m=1, \gamma=05$ ), correspond ing to fig. $2 \mathrm{Al}$, gives $\lambda \tau_{\text {RRKM }}=97$. This is clearly a case where trajectorles get trapped for periods long compared to the time required for equilibratıng the system or equivalently equipartitioning the system. Because the overall flow is ergodic, the system can be described by a statistical theory of reaction dynamics such as RRKM theory.

It is of interest to see what happens when the system is changed to make $\lambda \tau_{\mathrm{RRKM}}$ much smaller. This is easly accomplished by making the hole considerably larger $\mathrm{Fig} .2 \mathrm{Cl}$ gives the reactive flux $\bar{k}(t)$ for the system $\left(E_{0}=0.45, k=0.5, \gamma=0 \overline{5}\right)$. This system is dynamically identical to the system presented in fig. $2 \mathrm{Al}$, except that the relative width of the transltion state, $k=05$, is 25 tumes larger. The flux, $\bar{k}(t)$, now has fine structure. It exhibits decay over two widely separated time scales There is rapid decay in a time of order 1.4, a time corresponding to the tume it takes a typical actuvated trajectory startung at the hole to cross the width of a stadium and return to the hole. If the trajectory is still activated, that is, still sausfies the energy condition of $\dot{x}^{2}>2 E_{0}$, it will recross the TS and cease to contribute to the reactive flux until sucls tume that it can cross the hole again. The rapid decay is due to those trajectones that rapidly recross the TS. The rise around $t \approx 10$ is due to those trajectories that leave the well and then on the next collsion with the TS return. The long time decay, on the other hand, is due to those trajectories that emerge from the TS but are not sufficieritly activated when they collide with the TS. Because it takes a time on the order of $\lambda^{-1}$ to regan the energy to recross, these trajectories give rise to a slower decay. The long tume decay seems to be exponential, but the relative fluc- 
1

2

3
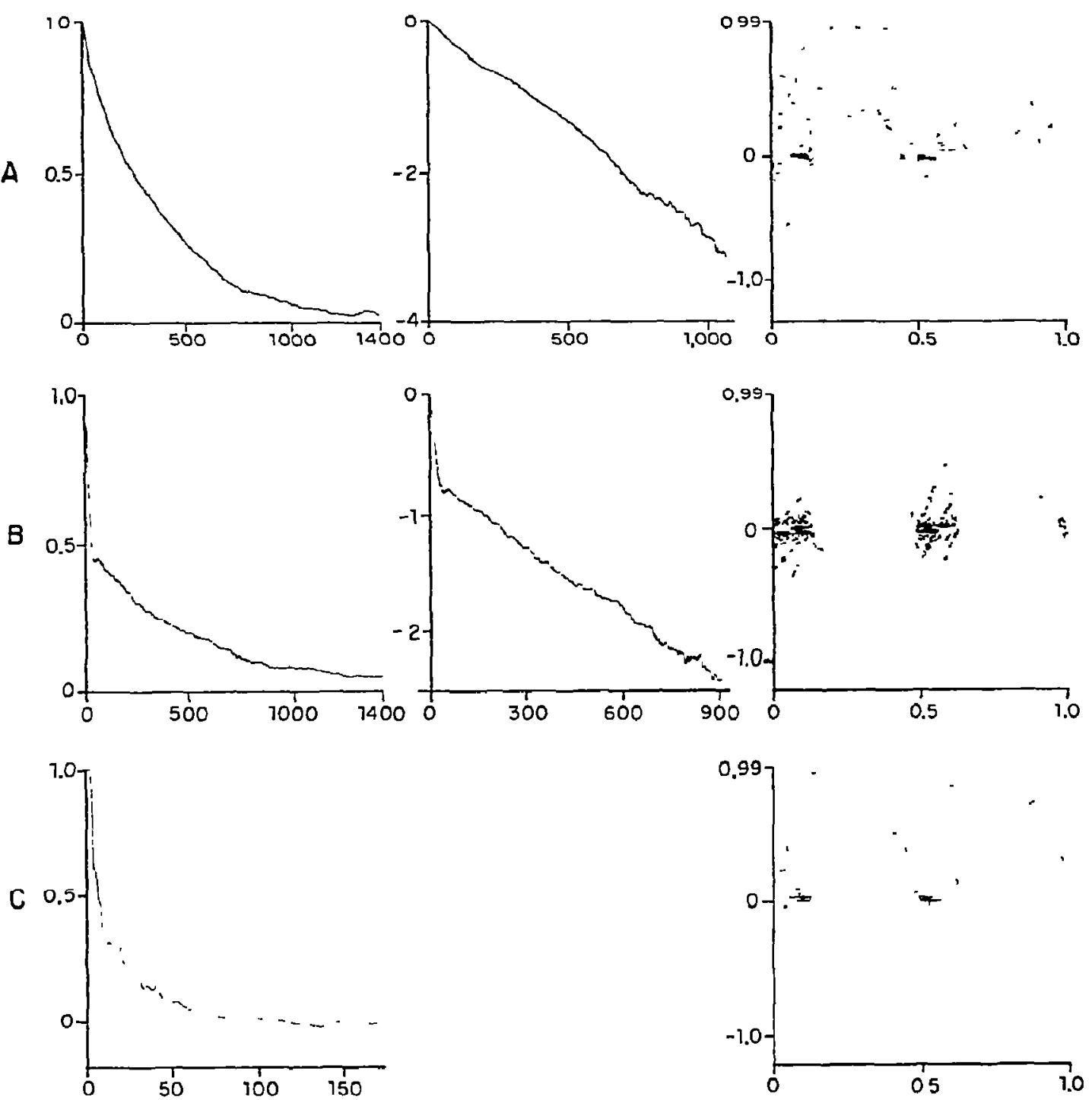

$\Gamma$ g. 2 Rows $A, B$ and $C$ correspond respectively to Stamese stadia with $\left(E_{0}=0.45, k=01, \gamma=05\right),\left(E_{0}=0.95, \kappa=0.1, \gamma=0.5\right)$ and $E_{0}=0.45, k=1,0, \gamma=0.5$ ), Columns 1,2 and 3 correspond respectively to the reactive flux, $k(t)$ [ $c f$ eq. (3)], (he logarithm, $-\ln k(r)$, and the short-time global sectson determuned by samplung the iniual states from eq. (6) and following the niotion for $a$ short tume. In A3 and B3, the trajectories were followed for 100 unuts, whereas in $\mathrm{C} 3$ they were followed for $\approx 15$ units The ord1nate and abscissa of the global section in column 3 are $\alpha$ and $\eta$ respectively (sec text prececding cq (6)) 
tuations are so large that they do not permit an accurate determunation of the rate constant. An approximate value is $\tau_{R \backslash n}^{-1} / \tau_{R R K i l}^{-1} \approx 0.5$. This should be noted along witl the fact that $\lambda \tau_{\text {RRKU }} \approx 4$.

It is clear that even when a system is completely stochastic, RRKM theory is not always valid. Thus Illustrates the requirement that the system must equipartition on a time scale fast compared to the tume on which $\mathrm{it}$ recrosses the transition state. A $K$ flow (which is a very strong property) does not necessarily satisfy thus requirement.

It is worth mentioning at thus point that there exists a measure preserving mappung of the stadium billiard that is analogous to the Poincare surface of section used to locate regular and irregular motion in continuous systems [3]. A trajectory in the stadium can be charactenzed by the positions $\eta$ at whech the billiard collides with the boundary, and the angles $\theta$ (or better $\sin \theta$ ) made by the velocity vector with the inward normal through the boundary at the point of impact. The collision position is defined uniquely by determining the distance one must travel clockwise froin some reference pount ( $\mathrm{Pl}$ in fig $1 \mathrm{~b}$ ) to the collision pount. $\eta$ is then defined as this distance divided by the length of the perimeter $|\Gamma|$ of the stadium. Any trajectory can then be mapped onto the carteslan space with $\alpha=\sin \theta$ as ordinate and $0 \leqslant \eta \leqslant 1$ as the abscissa This mapping was introduced by Bennettin and Strelcyn, who showed that the map is a global section. The stadium, being a $K$ flow, gives rise to a random map with a uniform distribution of points

Fig 2, column 3 gives maps made by following the trajectories contributing to the reactive flux Each trajectory is followed for the tume required for the llux to complete its fast decay The initial states are sampled from the normalized distribution

$f(r, p)=\frac{x^{2} 0(\dot{x}) \delta(x) \delta(E-H)}{\int \mathrm{d} \Gamma \dot{x} \theta(\dot{x}) \delta(x) \delta(E-H)}$.

Fig. 2A3 gives the short-time map of the trajectories used to compute fig. 2A1. The map looks perfectly uniform, except for trajectories corresponding to very sinall angles $\theta$. The high density of points at small values of $\sin \theta$ correspond to trajectones that bounce back and forth many tumes before reaching the caps of the stadium where they get randomized. The effect of these trajectones gives rise to a very small short- time decay in the reactive flux (not observable in the fig.). The short-time map (fig. 2C3) corresponding to fig. $2 \mathrm{Cl}$ is very similar to fig. $2 \mathrm{~A} 3$ sunce one is dealing with the same stadium.

The normalized reactive flux corresponding to $E_{0}$ $=0.95, \mathrm{k}=0.1$, and $\gamma=0.5$ is shown in fig. 2Bl. Fig. 2B2 gives the logaritlum versus tume. The long-time decay is seen to be exponential with rate constant $\tau_{\mathrm{R} \mathrm{n}}^{-1} / \tau_{\mathrm{RRKM}}^{-1}=049$. Interestingly $\lambda \tau_{\mathrm{RRKM}}=65$ for the system, so that on the face of it we expect RRKM theory to be valid. Why then does RRKM theory fall to describe reaction dynamics in this system?

Fig. 2B1 exhibits several important features. At very short times we observe several small oscillations in $\bar{k}(t)$ These occur on tıme scale $t=1.4$, and are due to trajectories of such small $\theta$ that they return to the hole in an activated state and can immediately recross several tumes The relative weight of these trajectories is given by the amplitude of the osclllations. This weight would grow if the hole size were made larger. A longer time decay, $t \approx 100$, is observed, and a very long tiune decay over a tume of order 1000 is observed. The short-time map generated by following the inttally sampled trajectories for a time $t \approx 100$ is shown in fig. 2B3. This map exhibits a very striking nonunformity of points. What is this higluly correlated short-time motion due to? Rernember over long periods of tume the nap should be unform'

This behavior can be attributed to the fact that for lugh barners, $E_{0}=0.95$, along the $x$ direction, the inttral set of trajectories lias velocity vectors pointung largely along the $x$ direction [the minimum value of $\bar{x}$ is $\left.\left(2 E_{0}\right)^{1 / 2}\right]$. This "cone" of trajectories widens as the barrier $E_{0}$ is decreased. Figs. 2B1-2B 3 corresponds to a very high barrier, $E_{0}=0.95$, and therefore gives nse to a velocity distrubution that is highly peaked in the forward direction ( $\operatorname{small} \operatorname{sm} \theta$ ). It is not difficult to show that a large fraction of these trajectones tend to get reflected back to the transition state with small $\sin \theta$ by the region of the semi-circle meeting with the two walls. In this case, because of the narrow velocity distribution, a stzeable fraction of the initial trajectories starting at the transition state continue to have small $\sin \theta$ for many collisions with the walls and retum to the transition state with small $\sin \theta$, recrossing it before spreading out over the energy surface in phase space. The remaining trajectories that do not coherently recross get trapped in the well for long 
penods of tume and escape through the hole at random times - hence the long-tume exponentral decay.

This mechanism explains the time decay of $\hat{k}(t)$ and the non-uniform feature of the short tume map. The clustering in the map occurs for small values of $\sin \theta$, and reflects the sampling of initial states with velocities peaki -1 in the forward direction, and the fact that snall values of $\sin \theta$ are maintaned by these trajectories over a long period of time. Since RRKM theory is expected to be valid only if equipartition can take place before recrossing, the relevant test is whether $\lambda \tau_{c}$ is large where $\tau_{c}$ is the time characterizing the fast decay. In the case corresponding to fig. $2 \mathrm{C} 1$, $\tau_{\mathrm{c}} \approx 100$ and $\lambda \tau_{\mathrm{c}} \approx 5$. Thus a large fraction of the trajectories recross the TS in a time short compared to $\lambda^{-1}$. Thus leads to the breakdown in RRKM theory. If the TS size is made small enough, the fraction that gets through is so scrercly reduced that RRKin is reestablished. We note parenthetically that it should be possible to estimate the mixing time $\tau_{M}$ for an initial sampling from [6]

$\tau_{\mathrm{M}} \approx(-\ln \mu) / \lambda$,

where this formula gives the tume required for a pount set of measure $\mu$ to cover plase space uniformly. For our purposes $\mu$ must be cliosen as the relative measure of unitial trajectones sampled by the distribution given in eq. (6). As the barner height increases, $\mu$ decreases, and $\tau_{M 1}$ gets larger. Then the condition for RRKM behavior is that

$$
\lambda \tau_{\text {RRKM }} /(-\ln \mu) \gg 1 \text {. }
$$

Fig. 3 summarizes our findings. The ordinate gives the ratio of $\tau_{\mathrm{R} i n}^{-1}$ to $\tau_{\mathrm{RRKM}}^{-1}$. Sunce in an ergodic system $\tau_{\mathrm{RRKM}}^{-1}$ is an upper bound on $\tau_{\mathrm{R} \times n}^{-1}$ the ordinate vanes between 0 and 1 . The abscissa is $\lambda \tau_{R R K M}$ Curve (A) corresponds to a series with $E_{0}=0.45, \gamma=$ 0.5 as a function of relative TS size, $k$. According to eq. (5) $\tau_{\text {RRKM }}$ increases as $K$ decreases. Since $\lambda$ is fixed, increasing $\lambda \tau_{\text {RRKM }}$ corresponds to decreasing hole size, and it is seen that $R\left(\equiv \tau_{\mathrm{R} \backslash \mathrm{n}}^{-1} / \tau_{\mathrm{RRKM}}^{-1}\right) \mathrm{in}$ creases monotonically with $\lambda \tau_{\text {RRKM }}$ reaching unity at which point RRKM theory is vald. All this means is that for small enough hole size equipartitioning takes place rapidly compared to recrossing of the hole. Curve (B) corresponds to a series with $\gamma=0.5, k=$ 0.1 , and $E_{0}$ varying between 0 and 0.95 . From eq. (5)

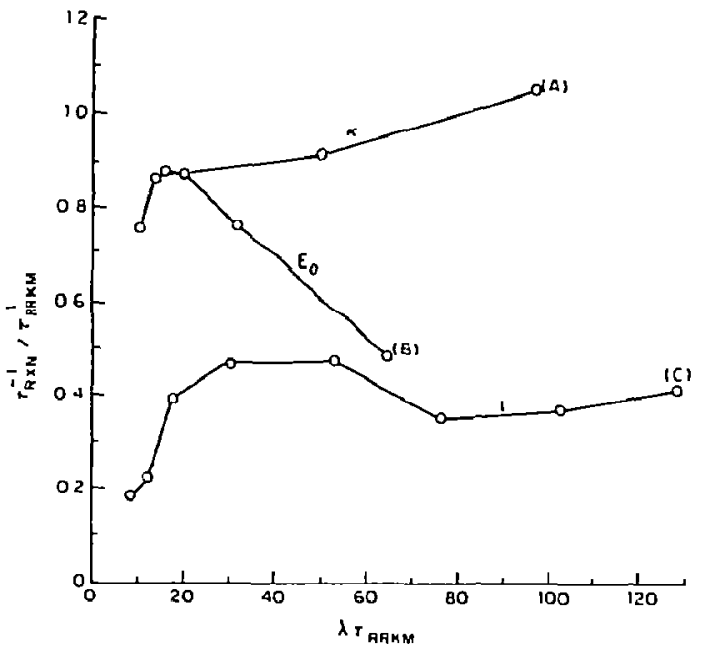

Fig. 3. A plot of the datho $R$ of the mleasured rate constunt to the RRKM rate constant, versus the Lyapunov exponent tImes the RRKM lifetime in a well, $\lambda_{\text {rRRKM }}$ Curve (A) corresponds to a series of Siamese stadal $\left(E_{0}=045, \gamma=0.5\right)$ with varuable $\kappa$ Curve (B) corresponds to a series of Siamese stadua with $(K=01, \gamma=05)$ with vareable $E_{0}$ vary ing from 0 to 095 Curve $(C)$ corresponds to a series of Siamese stadra with ( $a=02, E_{0}=095$ ) with varable $\gamma$ (by varying $\gamma \mathrm{dt}$ Constant $R=10$ )

it can be seen the $\tau_{\text {RRKM }}$ increases as $E_{0}$ increases. Since $k$ is constant for the system, tt follows that increasing $\lambda_{\tau_{R R K M} M}$ implies increasing $E_{0}$. Curve (B) shows that $R$ decreases monotonically with $\lambda_{\text {RRKM }}$ This is consistent with the foregong discussion which shows that as $E_{0}$ increases, the trojectories contributing to $\hat{k}(t)$ are dominated by those with $\operatorname{small} \sin \theta$, which can return from one collision with the "rando. mizing" cap of the stadıum with again a small $\sin \theta$ and thereby cross. Thus happens in a tume short compared to the equipartition time and leads to a nonstatistical rate constant. If $R$ is plot ted versus $\lambda \tau_{\mathrm{c}}$ or $\lambda \tau_{\mathrm{m}}$, we expect a monotonic increase to $R=1$. Curve (C) corresponds to a series at $E_{0}=0.95$, fixed hole size $a=0.2$, but variable $\gamma$. Here $\tau_{\mathrm{RRKM}}$ is fixed, but $\gamma$ is vaned (by varying $\gamma$ at constant $R=1$ ). From the work of Bennettin and Strelcyn [scaled by eq. (1)] we are able to determine the value of $\lambda$ and thereby $\lambda_{\text {RRKMI }}$. For small values of $\gamma$ (small $l$ ), $\lambda$ is smoll. As $\gamma$ is increased $\lambda \tau_{\text {RRKMI }}$ increases and we observe that $R$ grows, monotorucally. Because $E_{0}=0.95$ we never observe pure RRKM behavior. This follows 
from our previous discussion. The overall behavior of the curve is consistent with the view that over and above thus $E_{0}=0.95$ behavior, there is a dependence of the reaction dynamics and its associated rate constant on the tume required for equipartitioning compared to the time between recrossings, which is controlled by $\gamma$.

Thus we conclude that even in purely stochastic measure preservang flows it is still possible to find interesting dynamical correlations that invalidate the assumptions on which RRKM theory is based. It has been shown that it is possible to correlate the dynamic contributions to the rate constan: with the Lyapunov exponent. This correlation gives nse to the view that $\lambda$ can be regarded as analogous to the "collision rate" in collision theories of reaction dynamics.

\section{References}

[1] N. De Leon and B.] Berne, J. Chem Phys. 75 (1981) 3495.

[2] N. De Leon, R. Rosenberg and B.J. Berne, J. Phys Chem.. to be publushed

[3] R I Wolf and W L. Hase, J Chem. Phys 72 (1979) 316.

[4] W L Hase, Dynamics of molecular collisions, Part B, ed. W H. Niller (Plenum Press, New York, 1976)

[5] G. Bennetten and J.M.Strelcyn, Phys. Rev. A (1978) 773.

[6] I.A. Hamilton and $P$ Brummer, J. Phys Chem. 86 (1982) 2124.

[7] D Chandler, J. Chem. Phys. 68 (1978) 2954.

[8] N. De Leon and B.J. Bernc, Chem. Phys. Letters 93 (1982) 169.

[9] L.A. Bunimovich, Funkt. Analız. Jeg. Pılug 8 (1974) 73 [Funct. Anal. Appl 8 (1974) 254] 